\title{
Primórdios da analgesia de parto: a força das mulheres
}

\author{
The early history of labor analgesia: women's strength
}

\author{
Luciano Duarte Pires Pereira ${ }^{1}$ \\ ${ }^{1}$ Interno Complementar de Anestesiologia, Centro Hospitalar e Universitário de Coimbra, Portugal. \\ Este artigo é parte da Dissertação defendida pelo autor na Faculdade de Farmácia da Universidade de Coimbra, para obtenção do título \\ de Mestre em Farmacologia Aplicada.
}

\section{RESUMO}

Objetivos: Ao longo do tempo, as crenças, as posições religiosas e a comunidade médica marcaram a evolução da analgesia de parto. As mulheres, direta ou indiretamente, foram as grandes impulsionadoras deste importante marcador de igualdade social. O objetivo deste estudo é fazer uma revisão história sobre a evolução da analgesia de parto e determinar o papel da comunidade feminina nessa evolução.

Métodos: Revisão da literatura através da base de dados Pubmed e pesquisa de livros históricos sobre o tema.

Resultados: A analgesia de parto apresenta-se nos nossos dias como uma técnica segura e eficaz, tendo vindo a evoluir significativamente ao longo dos últimos 100 anos. As técnicas iniciais de anestesia/analgesia inalatória apresentavam riscos para a mãe e o feto, mas foram o início de um progresso demorado e necessário. Esta revisão faz uma síntese sobre os avanços e recuos da analgesia de parto e o papel da mulher nessa mudança.

Conclusões: A comunidade feminina teve um papel ativo e de pioneirismo no desenvolvimento da analgesia de parto. Instigadoras como a Rainha Victória ou investigadoras como Virginia Apgar, as mulheres são indissociáveis desse desenvolvimento.

DESCRITORES: ANALGESIA OBSTÉTRICA; HISTÓRIA DA MEDICINA; ANALGESIA EPIDURAL; ESCORE DE APGAR.

\section{ABSTRACT}

Aims: Over time, beliefs, religious views and the medical community marked the evolution of labor analgesia. Women, directly or indirectly, were major instigators of this important marker of social equality. The aim of this study is to make a historic revision on the evolution of labor analgesia and to determine the role of the female community on that evolution.

Methods: A review of literature through Pubmed database and research of historical books on the subject.

Results: Epidural analgesia is nowadays a safe and effective technique, having evolved significantly over the last hundred years. The initial inhalatory anesthesia / analgesia presented risks to the mother and fetus, but they were the beginning of a long and necessary evolution. This review provides an overview on the advances and retreats of labor analgesia and the role of women in these changes.

Conclusions: The female community had an active and pioneering role in labor analgesia development. Instigators like Queen Victoria or researchers as Virginia Apgar, women are inseparable from this development.

KEY WORDS: ANALGESIA, OBSTECTRICAL; HISTORY OF MEDICINE; ANALGESIA, EPIDURAL; APGAR SCORE. 


\section{INTRODUÇÃO}

Apesar do parto ser uma experiência feliz, a dor vivenciada pelas mulheres tem sido descrita como das mais intensas que as mulheres experimentam durante a vida. Num estudo recente, a dor do trabalho de parto, a oncológica, a do enfarte de miocárdio, a da cólica renal e a da queimadura, foram consideradas como as mais intensas. ${ }^{1}$ A dor do primeiro estágio do trabalho de parto origina-se no colo e segmentos uterinos inferiores e tem características viscerais, sendo sentida como uma dor silenciosa nas áreas correspondentes a T11 e T12. Com a progressão do parto, a dor torna-se mais intensa e é referida ao abdomen, zona lombar e sacro superior, áreas de T10 a L1. No segundo estágio, existe um componente somático associado a esta dor visceral, provocado pela distensão da vagina, da pelve e do períneo, assim como pela descida da cabeça fetal, que aumenta a pressão exercida na pelve. Essa dor é transmitida para entre a segunda e a quarta vértebra sacral.

As técnicas analgésicas atualmente disponíveis no trabalho de parto incluem terapias não farmacológicas, analgesia sistêmica, como perfusão de remifentanil, analgesia inalatória (mistura de protóxido e oxigênio, desflurano, sevoflurano) e técnicas loco-regionais. Entre estas últimas, a analgesia do neuro-eixo tem ganho preponderância, sendo actualmente considerada a técnica analgésica mais eficaz no trabalho de parto e com menos efeitos adversos na mãe e no feto. ${ }^{2}$

Nos Estados Unidos, $77 \%$ das parturientes recebem analgesia do neuro-eixo, ${ }^{3}$ e apesar da mortalidade materna ser de 7,5 por 100.000 nascidos-vivos, as complicações anestésicas são responsáveis por apenas $1,8 \%$ dessas mortes. ${ }^{4}$ Até se alcançar este nível de segurança percorreu-se um longo caminho durante mais de 100 anos. O objetivo deste estudo é revisar a evolução da analgesia do trabalho de parto, com um enfoque no papel que a mulher teve nesse progresso. Foram levantados os artigos sobre o tema na base de dados PubMed e consultados livros sobre história da Medicina.

\section{A ORIGEM}

Em 9 de Janeiro de 1847 James Young Simpson utilizou éter para anestesiar uma mulher com deformação pélvica, no trabalho de parto que estava em risco de morrer. ${ }^{5} \mathrm{~A}$ mãe sobreviveu a esse parto complicado sem dor, dando origem à analgesia em obstetrícia. Vários outros relatos se seguiram, na Inglaterra, Irlanda, França e Alemanha. ${ }^{6}$ Apesar do sucesso, obstetras proeminentes na Europa e América opuseram-se a este ato anestésico. Charles D. Meigs, Professor de Obstetrícia em Filadélfia, Estados Unidos, argumentava que a dor do parto tinha um objetivo, que era inseparável das contrações e que qualquer fármaco que anulasse a dor iria diminuir a força das contrações. ${ }^{7}$ Outros alegavam que a anestesia era perigosa para mãe e feto e que a dor era essencial para o reconhecimento de anormalidades no parto. ${ }^{8}$ Ramsbothan, do Hospital de Londres, Inglaterra, era mais radical e afirmava que a insensibilidade completa no parto através da administração de fármacos era uma fraude extremamente perigosa. ${ }^{6}$ Entre 1847 e 1848, Simpson fez 150 partos sob anestesia com éter e clorofórmio com os seguintes resultados: um natimorto em estado pútrido (morte intrauterina não relacionada com a anestesia), tendo os restantes bebês sobrevivido, todas as mães com alívio da dor. ${ }^{6}$ Como forma de convencer a comunidade internacional, em particular a americana, Simpson publicou vários trechos de cartas com resultados que tinha recebido de apoiantes da anestesia. Numa dessas cartas, o Professor Dubois, de Paris, afirmava que o éter anulava a dor das cirurgias obstétricas, que não destruía as contrações uterinas e dos músculos abdominais, que diminuía a resistência natural do períneo e que não parecia ter efeitos adversos na saúde do bebê. ${ }^{6}$

\section{O IMPULSO REAL}

Em 1853, a Rainha Victoria teve o parto do seu oitavo filho sob anestesia com clorofórmio, administrado por John Snow, o primeiro anestesista, um clínico meticuloso e respeitado em Londres. A comunidade médica continuava cética, a ponto de Thomas Wakley, fundador do periódico "The Lancet", considerar uma irresponsabilidade alguém ter aconselhado a administração de clorofórmio a sua majestade durante um parto perfeitamente natural. ${ }^{7}$ Os membros do Clero também se opuseram, mas por razões diferentes: a atitude da rainha não estava de acordo com os ensinamentos bíblicos. Não querendo uma confrontação pública, os médicos reais negaram que a rainha houvesse recebido anestesia. Porém estavam lançadas as bases para a separação do pensamento médico do religioso. Esse ceticismo contrastava com o entusiasmo que a analgesia de parto suscitava nas parturientes. As mulheres decidiam que queriam anestesia e impunham essa opção aos obstetras. Sobre isto, Simpson afirmava: "Os médicos homens podem opor-se à anestesia no parto mas será em vão; certamente que as nossas doentes irão forçar o seu uso, é uma questão de tempo."” 
Apesar de estarem lançadas as bases da analgesia de parto, os fármacos utilizados apresentavam riscos relacionados ao trabalho de parto, à criança e à mãe. ${ }^{8}$ Segundo alguns autores, esta seria a razão do ceticismo da maioria dos obstetras. ${ }^{9,10}$ Reconhecendo estes perigos, John Snow aconselhava o uso de éter devido ao menor risco de toxicidade, mas devia ser limitado ao segundo estágio do trabalho de parto ou a partos complicados. ${ }^{11} \mathrm{O}$ clorofórmio deveria ficar reservado para casos de dor severa pós-parto. ${ }^{12}$ A "Boston Society for Medical Improvement" concluiu que o éter era mais seguro que qualquer outro anestésico, que nenhum exigia tão poucas precauções no seu uso e que a única vantagem do clorofórmio era a de conveniência. ${ }^{13}$

\section{"TWILIGHT SLEEP"}

Durante o século XIX assistiu-se a uma mudança do paradigma da dor: esta perdeu a conotação teológica e tornou-se um processo biológico sujeito a estudo nos seus diversos tipos e controle por novos métodos da ciência e tecnologia. O alívio da dor (não apenas física) passou a ser visto como uma obrigação e uma necessidade social. Esta mudança surge associada a movimentos contra a escravatura, aos movimentos feministas, à revisão do sistema penal e à proteção de mulheres e crianças. ${ }^{10}$

No início do século XX surge a técnica "Twilight Sleep", inicialmente desenvolvida por von Steinbuchel e popularizada por Gauss em Friburgo, Alemanha. Consistia na administração de opioides, geralmente morfina $10 \mathrm{mg}$ e escopolamina em doses dependentes da resposta a um teste de memória. ${ }^{14}$ Tal como 50 anos antes, os obstetras opuseram-se a esse novo método. Após o trabalho do suiço Paul Zweifel, que em 1874 demonstrou a transferência placentária de fármacos, os obstetras estavam particularmente preocupados com o risco de depressão respiratória neonatal e asfixia. ${ }^{15}$ Posição oposta tiveram as parturientes: formaram a "National Twilight Sleep Association" que promovia e postulava o acesso à técnica a todas as mulheres que o desejassem. ${ }^{14}$ Esse entusiasmo durou até 1920, quando uma das principais apoiantes morreu durante $\mathrm{o}$ parto que decorria com "Twilight Sleep". ${ }^{7}$ No entanto, a técnica ficou associada a uma mudança da atitude conservadora em relação ao uso dos opioides no trabalho de parto.

\section{ANALGESIA REGIONAL E AGULHA TUOHY}

Em Julho de 1900 surge o primeiro relato de analgesia regional em obstetrícia. O suíço Oscar
Kreis utilizou cocaína intratecal em 6 parturientes com dilatação cervical completa. ${ }^{16}$ Nos 20 anos seguintes surgiram descrições de bloqueios lombares, paravertebrais, sacrais e peri-aórticos. Apesar do acesso ao espaço epidural através dos espaços interespinhosos ter sido descrito em 1906, apenas em 1921 a técnica foi aplicada, tornando-se popular 10 anos mais tarde. ${ }^{17} \mathrm{Em} \mathrm{1931,} \mathrm{Aburel} \mathrm{utilizou} \mathrm{a} \mathrm{técnica}$ de inserção de cateter através de agulha para produzir um bloqueio do plexo lombo-aórtico. ${ }^{17} \mathrm{Em}$ 1933, John Cleland demonstrou que um bloqueio paravertebral de T11 e T12 podia analgesiar a primeira fase do trabalho de parto. ${ }^{18}$ Os principais efeitos adversos nesses primeiros casos experimentais incluíam cefaleias pós-punção da dura, hipotensão e vômitos. O bloqueio epidural caudal contínuo foi introduzido em obstetrícia em 1941 por Higson e Edwards, ${ }^{17}$ tornandose muito popular e passando a ser a técnica de eleição em anestesia regional. Em 1945, Tuohy descreveu a agulha Tuohy, uma agulha 15 gauge com ponta de Huber, ou seja, ponta curva com orifício lateral. Em 1947, Curbelo combinou a agulha Tuohy com a técnica contínua, desenvolvendo a analgesia epidural contínua. ${ }^{19}$ A utilização crescente das técnicas por profissionais inexperientes e a percepção de que não era necessário monitorização levou a um aumento da taxa de complicações. Greenhill afirmava que o risco de morte não era o único inconveniente, sugerindo que a administração fosse feita apenas por anestesistas bem treinados em prevenir e tratar as complicações que pudessem surgir. ${ }^{20}$

Os resultados negativos com a anestesia regional levaram a um abandono da analgesia do trabalho de parto até 1950. Chalmers afirmava que o analgésico perfeito para o parto ainda estava para ser descoberto e que as objecções aos existentes levavam os médicos a evitar o seu uso. ${ }^{21}$ Por outro lado, a importância médica e social da dor mudou, voltando a ter propriedades fisiológicas. Esse período, que ficou conhecido como "Dark Age" da anestesia obstétrica, levou ao aparecimento da psicoprofilaxia e do parto natural. Este último resultou do movimento de reforma da saúde que postulava o regresso a um modo de vida mais natural como reparador da medicina materialista, argumentado que a vida saudável da mulher era a chave para um parto seguro..$^{22}$ Eugène Marais, naturalista sulafricano, afirmava que a dor é o estímulo do instinto maternal e, estando ausente, não existiria amor de mãe. Sustentava esta afirmação com experiências em ovelhas: o parto sob anestesia levava à recusa do animal em aceitar o filho, não acontecendo o mesmo no parto natural. ${ }^{23}$ 


\section{VIRGÍNIA APGAR}

Em 1949, numa conversa na cafeteria do Columbia University College of Physicians and Surgeons, Virginia Apgar descreveu o sistema de avaliação de recém-nascidos que viria a mudar a história da obstetrícia. ${ }^{24}$ Apenas publicado em 1953, o escore de Apgar teve três principais efeitos: substituiu a simples observação do recém-nascido, sujeita a ambiguidades, por uma pontuação reprodutível; forneceu critérios objetivos para iniciar a ressuscitação neonatal; e, mais importante, mudou o foco dos cuidados obstétricos. ${ }^{7}$ O critério primário de qualidade passou a ser o recémnascido, tornando-se uma medida sensível dos efeitos da analgesia no parto. Recém-nascidos de partos sob anestesia regional tinham escores de Apgar maiores do que os nascidos sob anestesia geral.

Nos anos seguintes, a analgesia epidural ganhou popularidade em razão da grande taxa de sucesso, ao início de ação rápido e ao fato do cateter ser colocado numa zona menos susceptível à infecção. ${ }^{16}$ Em função de novas exigências, foram aumentando os procedimentos de segurança. Os anestesistas começaram a procurar fármacos com menor efeito no feto e menor bloqueio motor, administração de dose teste que permitisse controlar a localização do cateter, doses fracionadas em pequenas bolus e concentrações de anestésicos locais mais baixas. ${ }^{9,25}$ Também a forma de administração mudou: em 1960 surgiram os cateteres plásticos maleáveis e em 1980 as bombas infusoras que permitem infusões exatas. Concomitantemente, houve alterações no sistema organizacional da saúde, com aumento dos padrões de qualidade e do número de médicos. Após 1970, a analgesia epidural tornou-se a técnica padrão, para a qual contribuiu a descoberta dos receptores opióides espinais. A combinação de opióides e anestésicos locais na analgesia epidural foi globalmente aceite e mantém-se nos dias de hoje como tratamento de eleição para a dor do trabalho de parto. ${ }^{9}$

\section{COMENTÁRIOS FINAIS}

A evolução da analgesia de parto reflete o estado social e o papel crescente da mulher na sociedade. Instigadoras como a Rainha Victória ou investigadoras como Virginia Apgar, as mulheres são indissociáveis desse desenvolvimento. O ceticismo inicial, as crenças religiosas, os movimentos feministas e o naturalismo foram fases que influenciaram a evolução da analgesia de parto. A comunidade feminina teve um papel ativo e de pioneirismo na instituição desse procedimento que permitiu o direito a um trabalho de parto livre de dor.

\section{REFERÊNCIAS}

1. Melzach R, Taenzer P, Feldman P, Kinch RA. Labour is still painful after prepared childbirth training. Can Med Assoc J. 1981;125:357-63.

2. Anim-somuah M, Smyth R, Howell CJ. Epidural versus non-epidural or no analgesia in labor. Cochrane Database Syst Rev. 2011;12:NoCD000331.

3. Cambic C, Wong C. Labour analgesia and obstetric outcomes. BJA. 2010;105:50-60.

4. Hawkins JL. Anesthesia-related maternal mortality. Clin Obstet Gynecol. 2003;46(3):679-87.

5. Bacon D. The Historical Narrative: Tales of Professionalism. Anesthesiol Clin. 2008;26:97-74.

6. Simpson J. On the early history and progress of anaesthetic midwifery. In: Simpson J. Anaesthetic Midwifery: report on its early history and progress. Edinburg: Sutherland \& Knox; 1848. p. 5-16.

7. Caton D. The History of obstetric anesthesia. In: Chestnut DH, Polley LS, Tsen LC, Wong CA. Obstetric Anesthesia Principles and Practice. 4th ed. Philadelphia: Mosby Elsevier; 2009. p. 3-12.

8. Caton D. Obstetric Anesthesia: the first ten years. Anesthesiology. 1970;33:102-9.

9. Caton D, Frolich M, Euliano T. Anesthesia for childbirth: controversy and change. Am J Obstet Gynecol. 2002;186(5):S25-30.

10. Caton D. John Snow's practice of obstetric anesthesia. Anesthesiology. 2000;92:247-52.

11. Cohen S. Strategies for labor pain relief - past, present and future. Acta Anaesth Scand. 1997;110:17-21.

12. Snow, J. Use of Chloroform. Lond J Med. 1849;1(1):50.

13. Boston Society for Medical Improvement. Report of a Committee of the Boston Society for Medical Improvement, On the Alleged Dangers which Accompany the Inhalation of the Vapor of Sulphuric Ether. Boston Med Surg J. 1861;65:229-54.

14. Caton D. In the present state of our knowledge - early use of opioids in obstetrics. Anesthesiology. 1995;82:779-84.

15. Caton D. Obstetric anesthesia and concepts of placental transport: a historical review of the nineteenth century. Anesthesiology. 1977;46:132-7.

16. Gogarten W, Aken H. A century of regional analgesia in obstetrics. Anesth Analg. 2000;91:773-5.

17. Foldes F, Colavincenzo J, Birch J. Epidural anesthesia: a reappraisal. Anesth Analg. 1956;35:33-7.

18. Chadwick H. Obstetric anesthesia - then and now. Minerva Anesthesiol. 2005;71:517-20. 
19. Martini J, Bacon D, Vasdev G. Edward Tuohy: the man, his needle and its place in obstetric analgesia. Reg Anesth Pain Med. 2002; 27(5):520-3.

20. Greenhill, J. Shall spinal anesthesia be used in obstetrics? Anesthesiology. 1950;11(3):283-8.

21. Chalmers J. Relief of pain in midwifery. BMJ. 1948;1(4552):661.

22. Moscucci O. Holistic obstetrics: the origins of natural childbirth in Britain. Postgrad Med J. 2003;79:168-73.

23. Hadfield J. Pain in childbirth. BMJ. 1948;1(4562):1155.

24. Calmes S. Virginia Apgar: at the forefront of obstetric anesthesia. ASA Newsletter. 1992;56:9-12.

25. Hong R. Less is more: the recent history of neuraxial labor analgesia. Am J Ther. 2010;17:492-7. C 